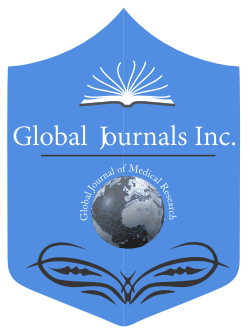

\title{
An Unusual Cause of Stridor after Decannulation of a Tracheostomised Patient
}

By Sailaja Kambhampati \& Meghana Yadav Abstract- Stridor is commonly seen post decannulation in a tracheostomised patient. Usually it occurs due to airway obstruction secondary to tracheal stenosis, granulation tissue, tracheomalacia. We report a rare case of stridor due to dynamic pharyngeal collapse after decannulation. A 68- year - old male who presented with inferior wall Ml and Complete Heart Block had to be put on a mechanical ventilator for hemodynamic instability and subsequently tracheostomised. Post decannulation he developed stridor and breathlessness. CT scan of neck revealed a supraglottic narrowing which on bronchoscopy showed a dynamic collapsibility of supraglottic area. This dynamic collapse was treated with non invasive positive pressure ventilation.

Keywords: stridor tracheostomy decannulation supraglottic narrowing dynamic pharyngeal collapse.

GJMR-D Classification: NLMC Code: WN 1

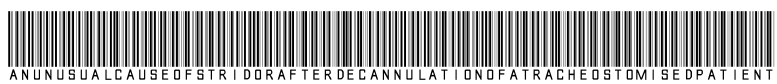

Strictly as per the compliance and regulations of:

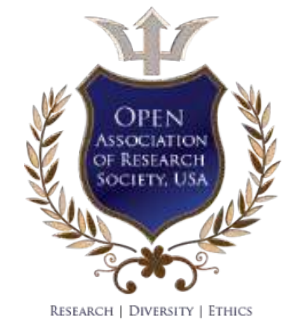

(C) 2020. Sailaja Kambhampati \& Meghana Yadav. This is a research/review paper, distributed under the terms of the Creative Commons Attribution-Noncommercial 3.0 Unported License http://creativecommons.org/licenses/by-nc/3.0/), permitting all noncommercial use, distribution, and reproduction in any medium, provided the original work is properly cited. 


\title{
An Unusual Cause of Stridor after Decannulation of a Tracheostomised Patient
}

\author{
Sailaja Kambhampati ${ }^{\alpha}$ \& Meghana Yadav ${ }^{\sigma}$
}

\begin{abstract}
Stridor is commonly seen post decannulation in a tracheostomised patient. Usually it occurs due to airway obstruction secondary to tracheal stenosis, granulation tissue, tracheomalacia. We report a rare case of stridor due to dynamic pharyngeal collapse after decannulation. A 68- year old male who presented with inferior wall $\mathrm{Ml}$ and Complete Heart Block had to be put on a mechanical ventilator for hemodynamic instability and subsequently tracheostomised. Post decannulation he developed stridor and breathlessness. CT scan of neck revealed a supraglottic narrowing which on bronchoscopy showed a dynamic collapsibility of supraglottic area. This dynamic collapse was treated with non invasive positive pressure ventilation.
\end{abstract}

Keywords: stridor tracheostomy decannulation supraglottic narrowing dynamic pharyngeal collapse.

\section{Case Report}

$\Lambda$ 68- year -old male was admitted to the hospital for Inferior wall Myocardial Infarction and Complete Heart block. He was a current smoker with 80 pack- years history and a hypertensive and diabetic on treatment. As he was hemodynamic ally unstable he had to be intubated after being taken up for
Percutaneous Coronary Angioplasty .As he had to be supported on the mechanical ventilator for a prolonged time with an endotracheal tube he had to be tracheostomised. In view of acute collapse of lung owing to thick tracheobronchial secretions, a good bronchial toileting was done. The patient was subsequently weaned off from the ventilator and then decannulated successfully. The follow-up visits in the OPD were uneventful clinically, but the patient was persistently complaining of progressive breathlessness and stridor. A CT scan of neck was advised and it showed supraglottic narrowing. Suspecting a laryngeal web, a video-assisted bronchoscopy was planned. Findings observed during bronchoscopy were dynamic collapsibility of the supraglottic area, with normal cords and no abnormality noted in the infraglottic area and tracheobronchial tree. The patient was advised nocturnal BIPAP. The patient's symptoms resolved completely and he returned to a normal lifestyle.

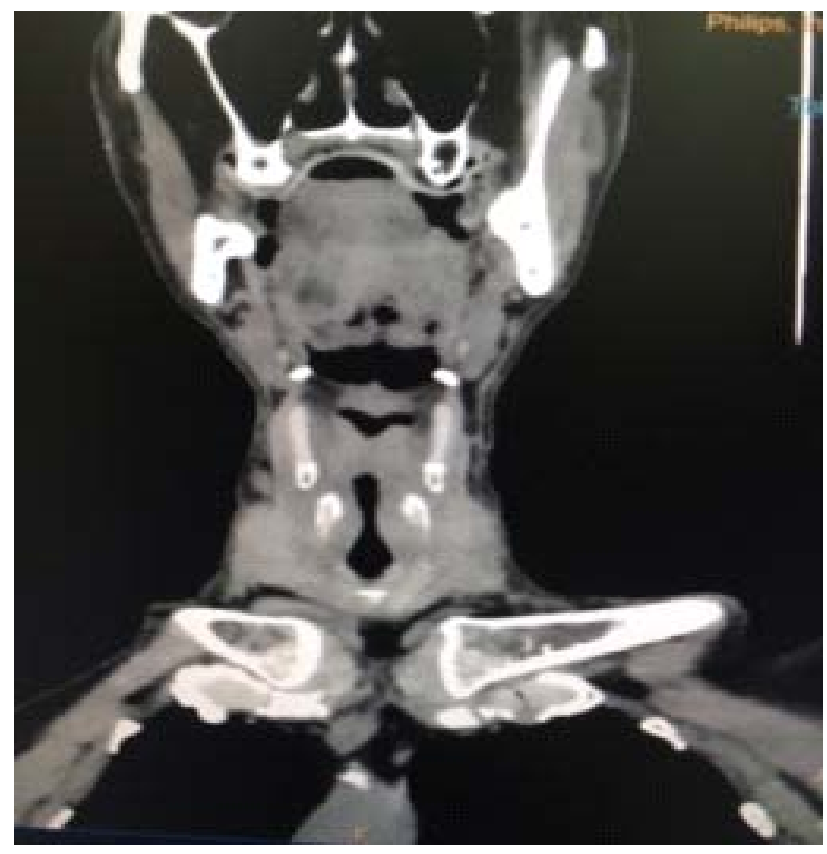

Figure 1: CT NECK coronal view showing supraglottic narrowing

Author: e-mail: ksailaja02@hotmail.com 


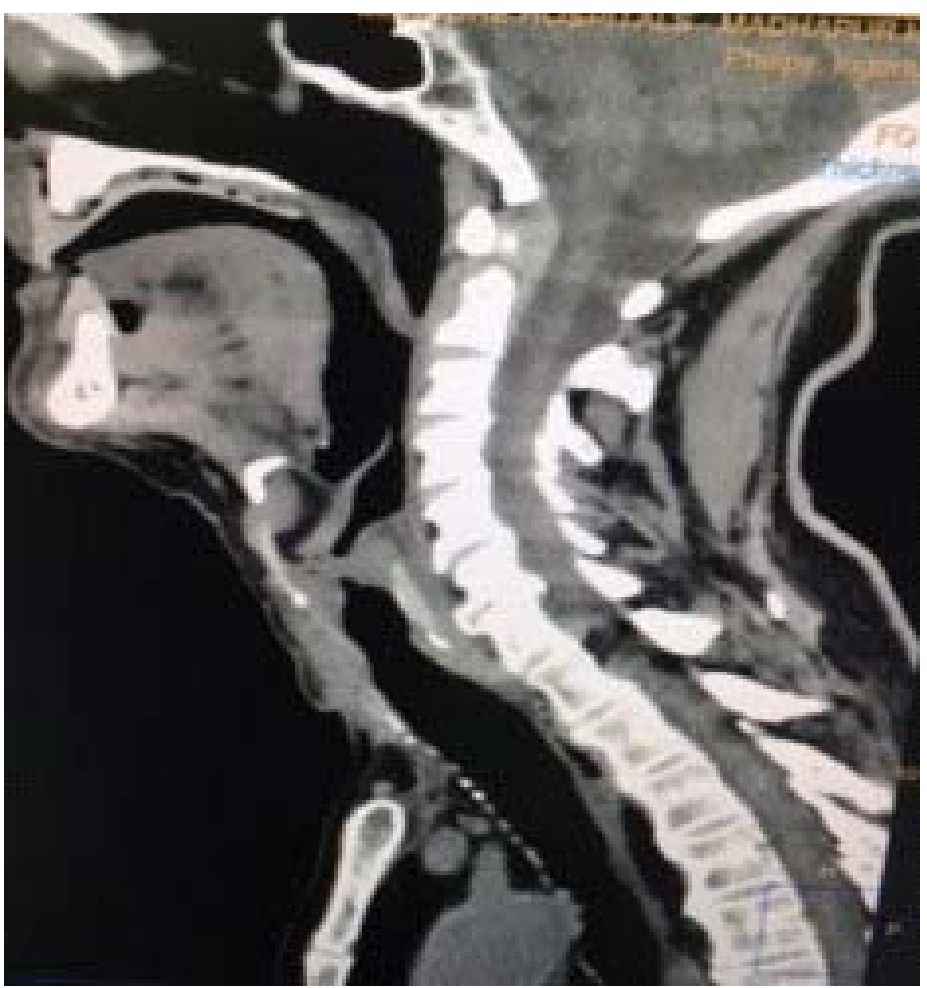

Figure 2: CT NECK sagittal view showing supraglottic narrowing

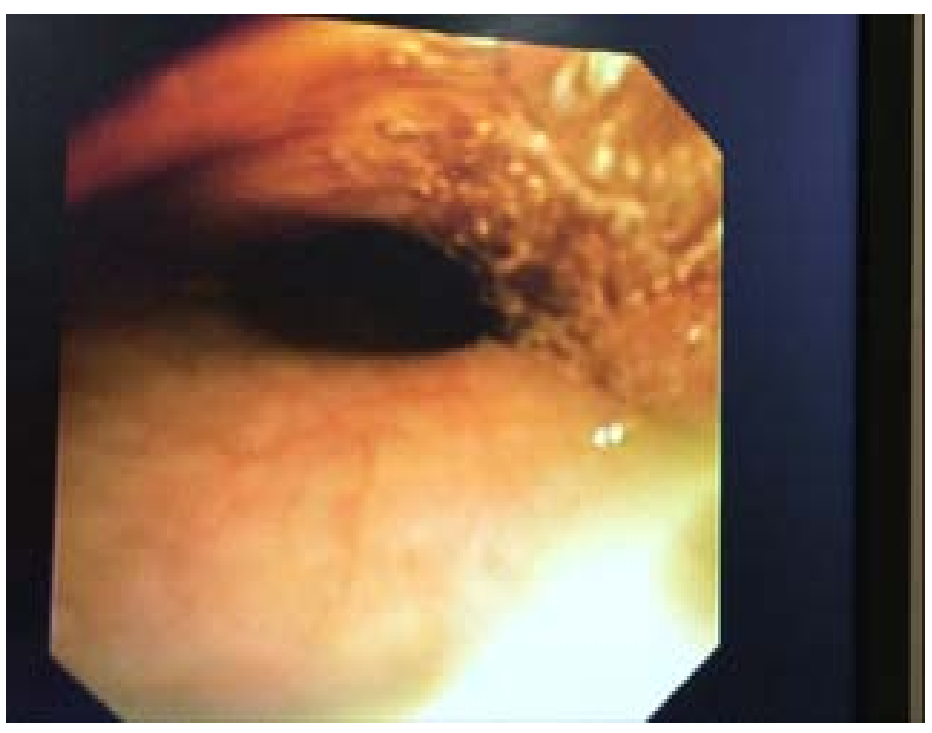

Figure 3: Bronchoscopy showing normal supraglottic airway 


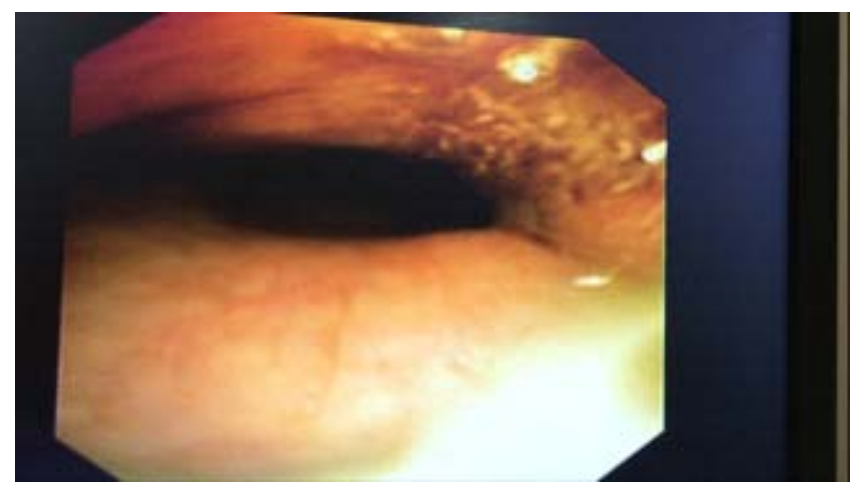

Figure 4: Bronchoscopy showing dynamic pharyngeal collapse

\section{Discussion}

Stridor in a post decannulated patient usually occurs due to tracheal stenosis, tracheomalacia, granulation tissue, nodules, polyps. The inicidence ranges from 20 to $67 \%$ in patients with long term tracheostomy tubes. But dynamic pharyngeal collapse may be an under recognized cause of stridor in post decannulated patient causing respiratory distress. Diagnosis is usually made by fiberoptic bronchoscopy. Treatment includes non invasive positive pressure ventilation.

\section{ili. Conclusion}

Even though stridor post decannulation is commonly due to tracheal stenosis, tracheomalacia dynamic pharyngeal collapsibility should also be considered as a potential cause. A video-assisted bronchoscopy aids in the detection of the dynamic collapse.

\section{References Références Referencias}

1. Late Complications of Tracheostomy Scott $\mathrm{K}$ Epstein Respiratory Care Apr 2005, 50 (4) 542-549

2. Chew J Y, Cantrell R W. Tracheostomy: Complications and Their Management. Arch Otolaryngol. 1972; 96(6): 538545. doi: 10.1001/archotol.1972.00770090816008

3. Mc Clelland R M. Complications of tracheostomy. $\mathrm{Br}$ Med J. 1965; 2(5461):567-569. doi:10.1136/bmj. 2. 5461.567

4. Norwood S, Vallina VL, Short K, Saigusa M, Fernandez LG, McLarty JW. Incidence of tracheal stenosis and other late complications after percutaneous tracheostomy. Ann Surg. 2000; 232 (2):233-241. doi:10.1097/00000658-20000800000014 